\title{
Detector Layout and Detection Probability Analysis for Physical Protection Systems of Nuclear Power Plants in Virtual Environments
}

\author{
Junbo Wang ${ }^{\mathrm{a}}$, Ming Yang ${ }^{\mathrm{b}}$, and Yuxin Zhang, ${ }^{\mathrm{a}, *}$ \\ ${ }^{a}$ College of Nuclear Science and Technology, Harbin Engineering University, Harbin, 150001, China \\ ${ }^{b}$ School of Electric Power, South China University of Technology, Guangzhou, 510000, China
}

\begin{abstract}
Based on the nuclear power plant environment and virtual reality engine Unreal Engine4, a simulation platform for physical protection systems is built in this paper. The virtual reality simulation method is used to simulate the layout of the detection and defense devices of the physical protection system of the nuclear power plant under the condition of the closest real environment. In order to improve the defense level of the physical protection system, the security system designer can be assisted to identify the weak links of the detection device layout scheme by analysing the effectiveness of the detection device.
\end{abstract}

Keywords: nuclear security; physical protection system; virtual reality; validity

(Submitted on December 21, 2018; Revised on January 23, 2019; Accepted on February 20, 2019)

(C) 2019 Totem Publisher, Inc. All rights reserved.

\section{Introduction}

At present, the design of physical protection systems of nuclear power plants in our country mainly adopts the EASI method of Sandia Laboratory. It is subjective and lacks systematic analysis. With the development of science and technology and the change of the security situation, it is difficult to update the system. In particular, the physical protection system personnel training and emergency planning evaluation cannot be effectively guided. In view of the fact that the design of physical protection systems of traditional nuclear power plants relies on expert experience, lacks visual design support, and has low design efficiency, the methods of autonomous layout of detection devices and physical barriers, human-computer interaction design, and evaluation of security control center are studied. In order to achieve the purpose of aiding physical protection system design, verification, and drilling training, human-computer interactive simulation and virtual drilling technology of physical protection systems are carried out in the environment of threedimensional models of nuclear power stations.

A physical protection system is a security defense system that uses physical barriers, detection delay technology, and human response ability to prevent theft, robbery, or illegal transfer of nuclear materials and destruction of nuclear facilities. Early detection of enemy intrusion is a prerequisite for obstruction and emergency response, which is essential to ensure the effectiveness of the physical protection system. In 1980, the International Atomic Energy Agency (IAEA) promulgated the Convention on the Physical Protection System of Nuclear Material [1], emphasizing the protection of nuclear materials in international transport and in the internal use, storage, and transport of nuclear materials. In 2010, it further promulgated the Nuclear Security Recommendations on the Physical Protection of Nuclear Material and Nuclear Facilities [2] to guide all countries to attach importance to the protection of nuclear materials and nuclear facilities.

Aiming at the phenomenon that the physical protection system design of the traditional nuclear power plant relies on expert experience, lacks visual design support, and has low design efficiency, this paper describes the realization of the independent layout function of detection devices and physical barriers under the 3D model environment of nuclear power plants, the design and verification of the auxiliary physical protection system, and drill training.

* Corresponding author.

E-mail address: yangm@scut.edu.cn 


\section{Nuclear Power Plant 3D Scene Construction}

Unreal Engine4 (UE4) is a virtual reality engine based on advanced dynamic lighting processing. It has dynamic and realtime rendering capabilities to simulate the physical properties of nuclear power plant equipment and changes in operating environments such as day and night and weather. The engine uses node connection to combine events, functions, and variables, which can describe complex logical relationships and simulate various behaviors and functions of system hardware and personnel. The engine, based on C++ program open source design, supports low-level development and provides visual programming functions to meet various technical requirements for application development. As shown in Figure 1, the three-dimensional scene construction of nuclear power plants using UE4 includes the following main steps:

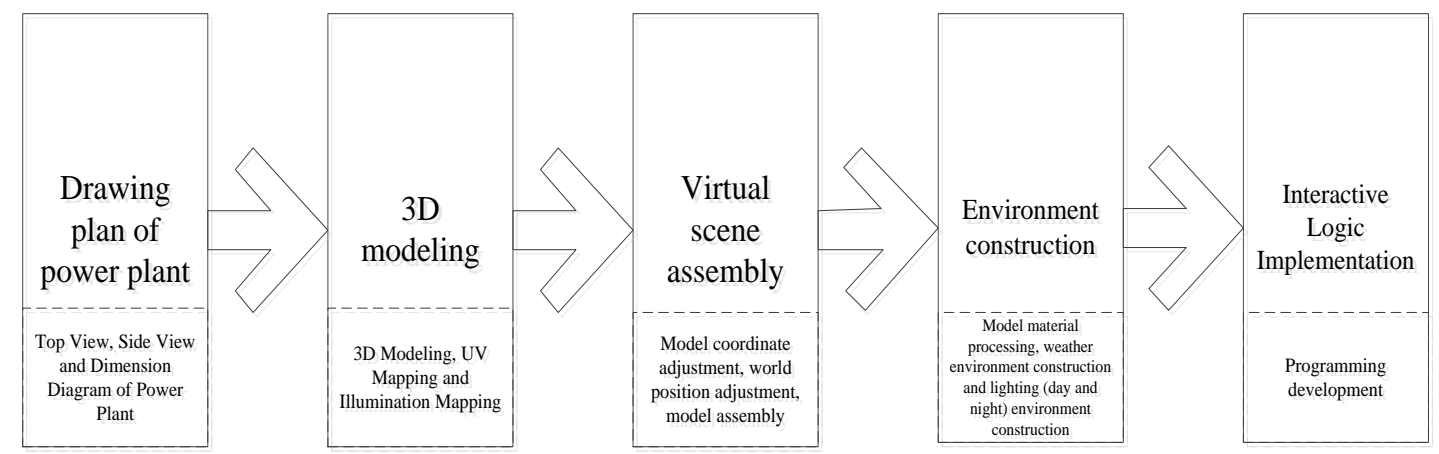

Figure 1. Plane graph of nuclear power plant

(1) Use CAD to draw a plan view of the nuclear power plant scene (Figure 2);

(2) Take the nuclear power plant scene plan as a reference, use 3DsMAX to make a 3D model of the nuclear power plant, and perform UV processing on the model;

(3) Introduce the 3D model of the nuclear power plant into the UE4 engine, and then assemble the power plant model according to the plan and make the plant environment construction;

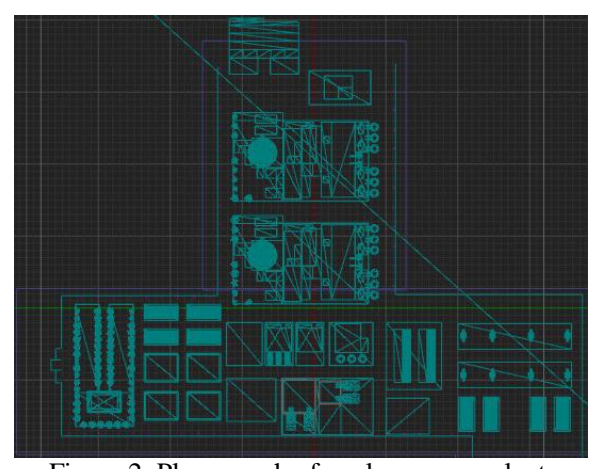

Figure 2. Plane graph of nuclear power plant

(4) Using the planar image processing tool to process the picture, import the picture resource into the engine, create the material, and assign it to the corresponding power plant model. The completed 3D rendering of the nuclear power plant and environment is shown in Figure 3.

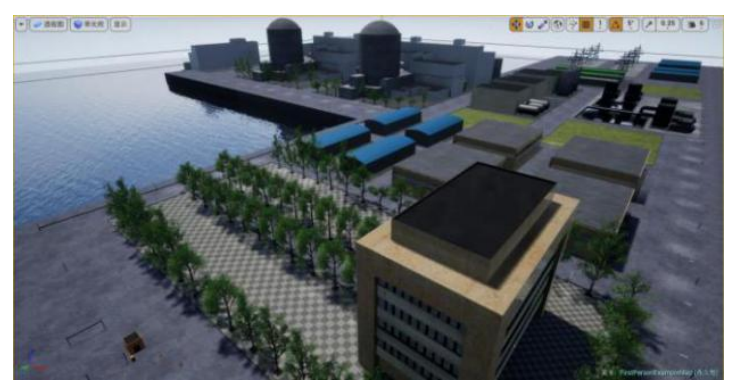

Figure 3. Plane graph of nuclear power plant 


\section{3D Design of Physical Protection System}

\subsection{Design Principles}

The physical protection system shall be designed in accordance with the three depth principles [3-5] of Defense in Depth, Protection in Depth, and Security in Depth. The following requirements must be met:

(1) In order to ensure the integrity, reliability, and effectiveness of the system, it is necessary to coordinate and improve the functions of physical protection system detection, delay, and response, so as to achieve an organic combination of civil air defense and technical defense measures;

(2) Set multiple physical barriers according to the facility level. Set up multi-level and different technology types of detection and alarm systems, in which the security protection levels of all parts of the same protection area are basically the same, with no obvious weak links and hidden dangers. Thus, defense in depth and protection in balance of the nuclear facilities are realized.

\subsection{Hierarchical Division}

As shown in Figure 4, the physical protection system is designed to divide the nuclear power plant into a control zone (ZS), a protected zone (ZP), and a critical zone (ZR) based on the importance of the protection target and the potential risk level. Different types of physical protection facilities are set according to the defense level requirements of different regions [6-8].

The control zone includes all areas that need protection from the physical protection system. The protection zone is located in the control zone, and the critical zone is located in the protection zone, including the nuclear island vital area, the joint pumping area (PX), and the security control center (UG) [9-10]. The functions and purposes of detection devices and defense devices are different among different levels. Physical protection system protection devices mainly include detection devices, defense devices, and delay devices. The detection capability of the detection device in the control area should be higher than that in the protected area and the key area, and the defense device delay capability in the key area should be higher than that in the control area and the protected area. The detection device is used to detect whether the adversary is invading or not. The collision body and visual field are added to all detection devices and detection devices in the virtual nuclear power plant environment. The different detection performance of the detection device can be analyzed. For example, the detection range, installation height, sensitivity, and other factors are analyzed. Delay devices, such as doors, windows, walls, and guardrails, are usually used to delay the enemy's invasion time, but they cannot be completely blocked. In the virtual reality platform constructed by the subject, all physical models are destructive, while defense devices and other obstacles have attributes that can be bypassed or crossed.

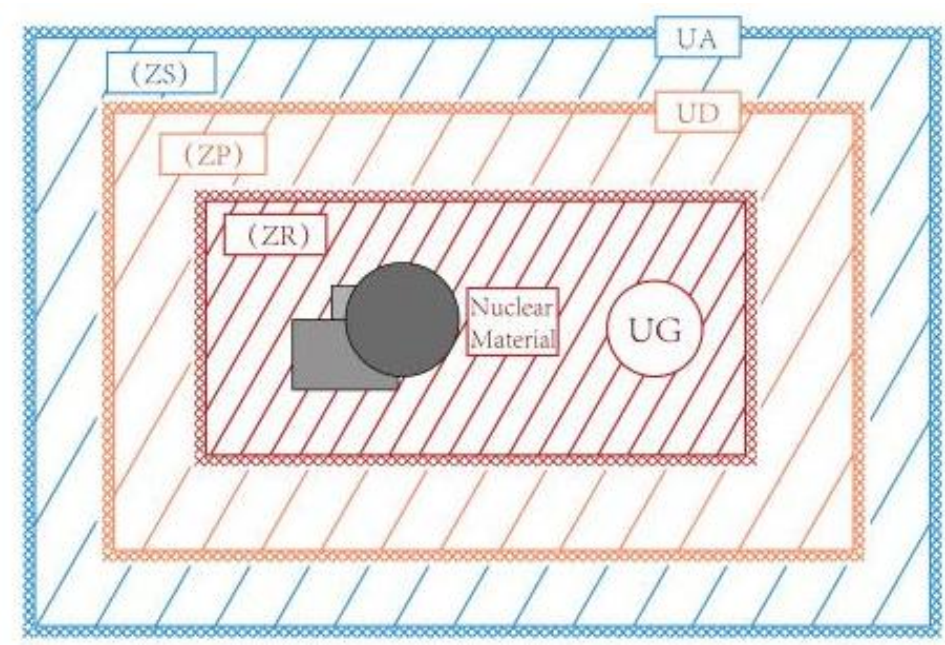

Figure 4. Safety zone division of nuclear power plant

\subsection{D Design}

According to the design principles and hierarchical division of the physical protection system, the physical protection 
system defense device arrangement is realized by the UE4 engine visual programming function based on the 3D scene of the nuclear power plant, which is divided into the following steps:

(1) Set the corresponding attributes of the protection device, such as the height of the physical barrier, the length of the blind area of the detection device and the environmental impact, etc.

(2) Set different environmental factors, such as day and night, weather, etc.

(3) Add virtual characters (security personnel or intruders) in the 3D scene of the nuclear power plant, and set different action modes, such as walking, running, crawling (climbing), jumping, etc.

(4) Add a detecting device to the scene by controlling the virtual character.

The control zone adopts a single-layer barbed wire fence as the physical perimeter, and the main entrance and exit is provided with triangular gates, gates, and electric retractable doors for the access control of the personnel and vehicles.

The physical barrier of the protected zone is composed of a double-layered barbed wire fence unit for isolation. It is equipped with alarm detectors such as microwave, Doppler, vibrating cable, and closed-circuit television cameras to achieve full coverage intrusion detection and video surveillance of the perimeter of the protected area. The main entrance and exit of the protected area is equipped with double revolving door control, and the passage of personnel and vehicles is controlled by the use of gates, hydraulic barriers, and electric retractable doors.

In the critical area, the inner perimeter fence and the outer lightweight barrier fence form the perimeter of the entity, and a monitoring zone is set up between the fences. A tension wire detector and a closed-circuit television camera are arranged on the inner fence to realize full coverage intrusion detection and video monitoring of the critical perimeter. In principle, the vehicle is not allowed to enter the critical zone, and the personnel entering and leaving the critical zone have to pass through the two revolving doors of the critical zone to be controlled.

The Defense Control Center is located in the protected zone and is protected as an independent vital area. The physical barrier of the Defense Control Center consists of a reinforced concrete wall of its building. The personnel entering the Defense Control Center must use access cards to pass through the pneumatic gate on the first floor. The access to the room on the second floor of the center is also equipped with a personnel pneumatic gate, with a two-channel electronic control door lock controlled by both the card and palm print readers. Afterwards, the personnel entering the room of the center are also required to confirm in front of the monitor before the security guard manually turns on the switch of the electromagnetic door to let them in.

\section{Detecting Device Layout Optimization based on Detection Probability Analysis}

Detection is the premise of ensuring the delay and response function of the physical protection system. Failure of detection will directly lead to degradation and even failure of the protection device delay and response function [11].

The SAVI model is the most common method for intrusion path analysis and vulnerability detection of physical protection systems. This method uses a one-dimensional model to analyze the effectiveness of physical protection systems, and it uses cumulative detection probability $P_{D}$ and residual intrusion time $T_{R}$ to determine key detection points. The formula is as follows:

$$
\left\{\begin{array}{l}
P_{D}=1-\prod_{i=1}^{m} P_{D-i}^{\prime} \\
T_{R}=\sum_{j=m}^{n} T_{j}>T_{G}
\end{array}\right.
$$

$P_{D-i}^{\prime}$ : From the beginning of the invasion, the detection point of layer $i$ does not detect the probability of enemy invasion; $T_{R}$ : the minimum delay time, or the remaining invasion time of the enemy; $T_{j}$ : the $j^{\text {th }}$ defense device delays the invasion time of the enemy; $T_{G}$ : the response time of the defense personnel; $m:$ the number of detection points. 
However, there are three problems in the SAVI model:

SAVI does not consider the distance between the protective devices on each protective layer; it only allows for the shortest distance or a reasonable distance between the input protective layers;

SAVI adopts a conservative calculation method for the shortest time for each protection device to be successfully handled by the opponent and the lowest probability of detecting the opponent, without considering the connection of the number of these protection devices.

Protection devices in physical protection systems are relatively complex, and SAVI model does not describe the intrusion strategy and defense strategy in detail.

Therefore, this paper combines the intrusion behavior with the layout of detection devices to further enhance the effectiveness of physical protection system design by virtual simulation combined with three-dimensional scenes.

The detection system includes sensors for detecting external and internal intrusions, video surveillance, admission control, and alarm communication systems. The detection probability of the detection device is affected by factors such as the intrusion mode of the detection target, the environment, and the sensitivity of the sensor.

In addition to the specified detection function, the detection device layout should also use redundant replacement and diversified means to improve the reliability of the detection function [12]. At the same time, the number of detection devices should be reduced and the economy should be improved [13-14].

This paper proposes a probabilistic based detection device layout method, and the steps are as follows:

(1) Detection range coverage: As shown in Figure 5, the control area, the protection area, and the critical area are divided by a square grid with a side length of $\mathrm{m}$. In order to avoid the formation of a detection blind zone in the grid, the mesh side length is $m \leq \min \left\{D_{1}, D_{2}, \cdots, D_{n}\right\}$, in which $D_{i}$ is the detection range of the $i$ type of detecting device.

(2) Detection area constraint analysis: For each grid, a detection probability correction factor $\beta_{i}\left(0 \leq \beta_{i} \leq 1\right)$ is defined according to the type of detector that cannot detect the grid, wherein $\beta_{i}=0$ means the area where the grid is located cannot be detected by the $i$ type detecting device, $\beta_{i}=1$ means the area where the grid is located can be non-destructively detected by the $i$ type detecting device, and $0<\beta_{i}<1$ indicates that the area where the grid is located has an obstructive effect on the $i$ type detecting device.

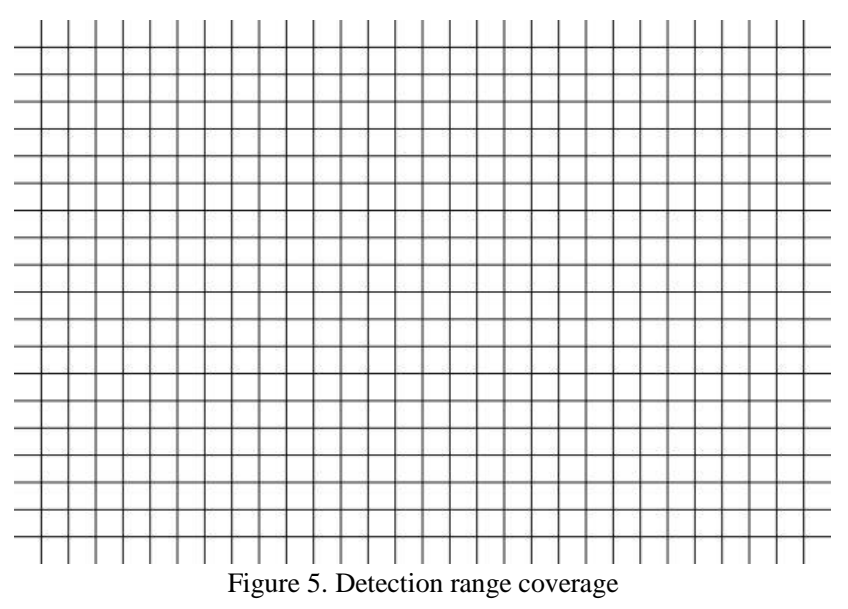

(3) Installation of the detecting device: The detecting device layout is performed according to the detection requirements of the protection object (for example, the entrance and exit) and the detection range of the detecting device.

(4) Probing probability analysis: Calculate the probability that the grid is detected by the detecting device. Figure 6 is a graph showing the relationship between the detection capabilities of several different types of detection devices over a 
certain detection range and the detection distance over a single axis. Different types of detection devices are affected by the environmental impact factor $E$ and the detection object ability $M$ in addition to the detection distance. Equation (2) is the average probability that each grid is detected by the detection device within the power plant range, where $x_{1}, x_{2}, y_{1}, y_{2}$ is the plane coordinate position of the detection range.

$$
\overline{P\left(x, y, M_{i}, E\right)}=\frac{\int_{x_{1}}^{x_{2}} \int_{y_{1}}^{y_{2}} P\left(x, y, z, M_{i}, E\right) \mathrm{d} x \mathrm{~d} y}{\left(x_{2}-x_{1}\right)\left(y_{2}-y_{1}\right)}
$$

Within the sensor detection range of each subordinate, the probability of each grid being detected is:

$$
P\left(x, y, M_{i}, E\right)=1-\prod_{\forall i}\left[1-\overline{P_{i}\left(x, y, z, M_{i}, E\right)} \times \beta_{i}\right]
$$
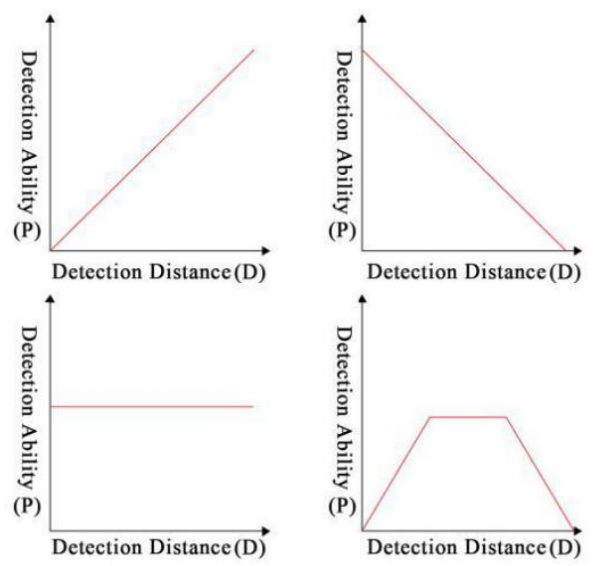

Figure 6. The relationship between sensor detection ability and detection distance

(5) Redundant design: Due to the limitations of the detection device and the influence of environmental factors, the detection device of different types, different specifications, and even different manufacturers should be adopted for redundant design. The failure of the detection device is set one by one, and the change of the detection effect of the grid is calculated and observed, so as to avoid a single failure of the detection device, resulting in insufficient detection capability and even a blind area.

(6) Optimizing the layout of the detection device: Adjust the position and type of the detection device, compare and analyze the comprehensive economy and effectiveness, and determine the suitable layout scheme of the detection device.

Figure 7 is a grid space detection probability distribution when a plurality of detecting devices is arranged in a 3D scene.

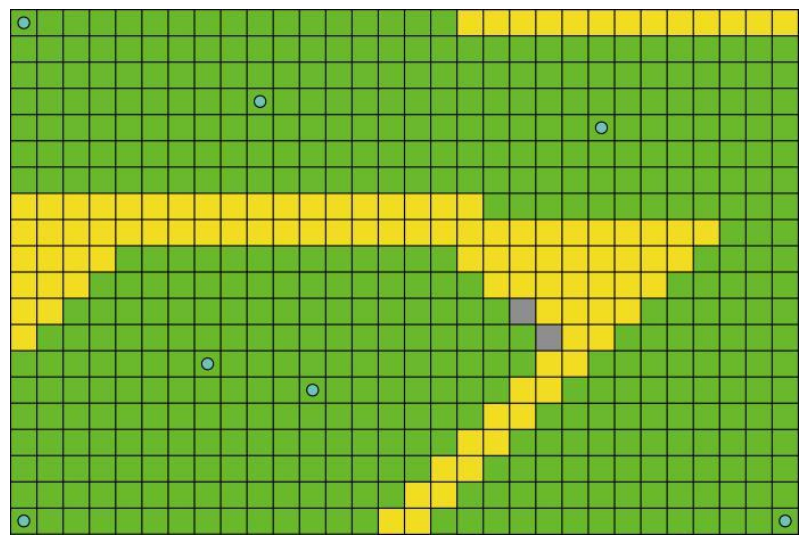

Figure 7. Detection probability after optimizing the layout of detectors 
As shown in Equation (4), according to the probabilistic requirements of the detection technique $P_{s}$, in the visual environment, the probability of detecting the grid is divided into three levels, wherein:

Level 1: The probability of detection meets the technical specifications and is indicated in green.

Level 2: The detected probability is lower than the technical specification and is indicated in yellow.

Level 3: Cannot be detected, indicated in gray.

$$
P(x, y, E)=\left\{\begin{array}{l}
1, P(x, y, E) \geq P_{s} \\
2,0<P(x, y, E)<P_{s} \\
3, P(x, y, E)=0
\end{array}\right.
$$

According to the design requirements of the physical protection system and the layout method of the detection device, the layout of the physical protection system detection device in the 3D scene is optimized. Finally, the probability of detecting the spatial grid of the nuclear power plant is shown in Figure 8.

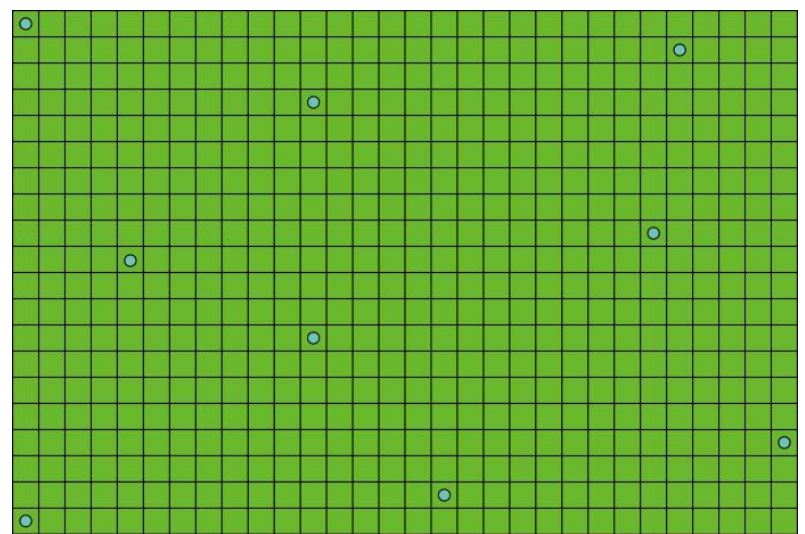

Figure 8. Detection probability after optimizing the detection device layout scheme

Considering the influencing factors of the height direction $Z$, each cell is a cube with a side length of $m$, the plane analysis is extended to spatial analysis, and the average detection probability calculation formula is corrected from Equations (2) to (5). Among them, $x_{1}, x_{2}, y_{1}, y_{2}, z_{1}, z_{2}$ is the spatial coordinate position of the detection range. The formula takes into account the detection range of the detection device in the $3 \mathrm{D}$ space of the entire power plant, avoiding the detection blind zone in the height space. It can evaluate the effectiveness of detecting low-altitude intrusion, but it requires a large amount of statistical work.

$$
\overline{P\left(x, y, z, M_{i}, E\right)}=\frac{\int_{x_{1}}^{x_{2}} \int_{y_{1}}^{y_{2}} \int_{z_{1}}^{z_{2}} P\left(x, y, z, M_{i}, E\right) \mathrm{d} x \mathrm{~d} y \mathrm{~d} z}{\left(x_{2}-x_{1}\right)\left(y_{2}-y_{1}\right)\left(z_{2}-z_{1}\right)}
$$

\section{Conclusions}

Physical protection systems play an important role in the whole life cycle of nuclear power plant construction, operation, and decommissioning. The planning of the system must refer to the design of nuclear power plants. With the progress of science and technology and the change of the national safety environment, the design of nuclear power stations must be improved constantly. Therefore, this study suggests developing an integrated design and analysis platform system of physical protection systems, which can be used to assist the physical protection system design, division, analysis, and evaluation.

This paper uses the UE4 engine to design physical protection systems in the virtual plant environments of nuclear power plants, which can visually reproduce the composition and operation of the nuclear power plant physical protection system to the greatest extent. It can further combine the detection device effectiveness analysis technology to realize the 
visualization of the detection effect, effectively helping security system designers identify the vulnerability and weak links in the detection device layout to facilitate the design change and optimization of the physical protection system.

In addition, the technology proposed in the paper also provides a technical basis for security drills and training in the virtual environment. Combined with the intrusion path identification and defense effectiveness evaluation functions, the physical protection system and the emergency response program effectiveness evaluation function can be realized, which are of great significance to the reduction of design cost and the improvement of the defense level of physical protection systems.

\section{Acknowledgements}

My deepest gratitude goes first and foremost to Professor Yang Ming, my supervisor, for her constant encouragement and guidance. He walked me through all the stages of the writing of this thesis. Without his consistent and illuminating instruction, this thesis could not have reached its present form.

\section{References}

1. D. L. Siazon Jr, "The Convention on the Physical Protection of Nuclear Material," IAEA BULLETIN, Vol. 22, No. 3-4, pp. 5762,1980

2. "Nuclear Security Recommendations on Physical Protection of Nuclear Material and Nuclear Facilities," International Atomic Energy Agency (IAEA), Vienna, 2010

3. C. L. Smith, "Understanding Concepts in the Defence in Depth Strategy," in Proceedings of IEEE 37th Annual 2003 International Carnahan Conference on Security Technology, pp. 8-16, October 2003

4. V. R. Nunes, L. Steven, and J. Ciuk, "A More Rigorous Framework for Security-in-Depth,” Journal of Applied Security Research, Vol. 6, No. 3, pp. 372-393, 2011

5. M. Coole, J. Corkill, and A. Woodward, "Defence in Depth, Protection in Depth and Security in Depth: A Comparative Analysis Towards a Common Usage Language," in Proceedings of Australian Security and Intelligence Conference, pp. 27-35, 2012

6. M. L. Garcia, "Vulnerability Assessment of Physical Protection Systems," Elsevier, 2005

7. H. A. Bennett, "EASI Approach to Physical Security Evaluation," Sandia Lab. Albuquerque, NM, USA, Tech. Rep. SAND-760500, 1977

8. R. A. AI-Ayat, T. D. Cousins, and E. R. Hoover, "ASSESS Update-Current Status and Future Developments," Lawrence Livermore National Laboratory, 1990

9. M. J. Hicks, M. S. Snell, J. S. Sandoval, and C. S. Potter, "Physical Protection Systems-Cost and Performance Analysis: A Case Study," IEEE Aerospace and Electronic Systems Magazine, Vol. 14, No. 4, pp. 9-13, April 1999

10. S. S. Jang, S. W. Kwak, H. S. Yoo, J. S. Kim, and W. K. Yoon, "The Tile-Map based Vulnerability Assessment Code of a Physical Protection System: SAPE (Systematic Analysis of Protection Effectiveness)," 2008

11. Z. Bowen, "Research on the Effectiveness Analysis Technology of Nuclear Power Plant Physical Protection System in Virtual Environment," Harbin Engineering University, Harbin, 2018

12. P. Byrne, "Application Firewalls in a Defence-in-Depth Design," Network Security, Vol. 2006, No. 9, pp. 9-11, 2006

13. R. Nunes-Vaz, S. Lord, and J. Ciuk, "A More Rigorous Framework for Security-in-Depth," Journal of Applied Security Research, Vol. 6, No. 3, pp. 372-393, 2011

14. P. U. Union, "Monitoring for Radioactive Material in International Mail Transported by Public Postal Operators," Reference Manual, 2006 